\title{
Desprovincializar las universidades europeas
}

Déprovincialiser les universités européennes

Deprovincializing european universities

\author{
Caroline Rolland-Diamond, Capucine Boidin, James Cohen y Ramon \\ Grosfoguel
}

Traductor: Marta Gómez

\section{OpenEdition}

1 Journals

Edición electrónica

URL: https://journals.openedition.org/ideas/3053

DOI: 10.4000/ideas.3053

ISSN: 1950-5701

Este artículo es una traducción de:

Déprovincialiser les universités européennes - URL : https://journals.openedition.org/ideas/278 [fr]

Otras traducciones del artículo:

Deprovincializing European universities - URL : https://journals.openedition.org/ideas/2292 [en] Desprovincializar as universidades europeias - URL : https://journals.openedition.org/ideas/3048 [pt]

Editor

Institut des Amériques

Referencia electrónica

Caroline Rolland-Diamond, Capucine Boidin, James Cohen y Ramon Grosfoguel, «Desprovincializar las universidades europeas», IdeAs [En línea], 2 | 2012, Publicado el 08 febrero 2012, consultado el 19 octubre 2022. URL: http://journals.openedition.org/ideas/3053; DOI: https://doi.org/10.4000/ideas. 3053

Este documento fue generado automáticamente el 19 octubre 2022.

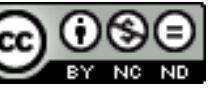

Creative Commons - Atribución-NoComercial-SinDerivadas 4.0 Internacional - CC BY-NC-ND 4.0 https://creativecommons.org/licenses/by-nc-nd/4.0/ 


\title{
Desprovincializar las universidades europeas
}

\author{
Déprovincialiser les universités européennes \\ Deprovincializing european universities
}

Caroline Rolland-Diamond, Capucine Boidin, James Cohen y Ramon
Grosfoguel

Tradución : Marta Gómez

\section{Las universidades europeas en una encrucijada}

La clasificación de Shanghái publicada en 2003 hirió profundamente el orgullo francés al relegar a sus universidades y grandes escuelas a los últimos puestos del palmarés, muy por detrás de las universidades de las megalópolis norteamericanas, británicas y japonesas. Numerosas críticas rechazaron esta clasificación. Las más importantes probablemente tenían que ver con la ventaja comparativa de la que gozan las publicaciones en lengua inglesa en la competición internacional y con la dificultad de jerarquizar las universidades en el ámbito de las ciencias humanas y sociales. El análisis actualizado de varias clasificaciones mundiales efectuado por Hervé Théry para la sección Enfoques de la revista invita en primer lugar a descentrar nuestra mirada: «En las cuatro clasificaciones seleccionadas, la concentración de universidades clasificadas en algunas regiones del mundo es tal que ha sido necesario añadir al planisferio tres zooms para ampliar la situación de Estados Unidos, Europa y Japón». El presente dossier invita al lector francófono a ampliar sus temas de preocupación, más allá del «declive» o del «carácter incomparable» del modelo francés, a las desigualdades mundiales ${ }^{1}$, en particular al desequilibrio existente entre las universidades norte y sudamericanas aun cuando ciertas universidades brasileñas, mexicanas, argentinas y chilenas aparecen en algunas clasificaciones ${ }^{2}$.

Habría que utilizar las clasificaciones como la sal, evitando el exceso de celo y el rechazo estéril, por retomar la expresión de Boaventura de Sousa de quien los lectores podrán encontrar reflexiones y propuestas contundentes para las universidades 
europeas en la sección Enfoques de este número. El objetivo general de este dossier es reflexionar sobre la situación de las universidades europeas tomando como punto de referencia la experiencia americana. La influencia del modelo americano de educación superior se hizo sentir primero en América Latina antes de llegar a Europa. La liberalización del mercado de la educación superior, la multiplicación de las agencias de evaluación, además de la creación de instituciones universitarias especializadas para ciertas minorías, empiezan a ser una realidad en América Latina a partir de los años 90. El artículo de Manuela Boatcă publicado en este dossier muestra precisamente cómo las «iniciativas de excelencia» en Alemania son ideadas como un medio para ponerse a la altura del nuevo occidente: el modelo estadounidense de educación superior. Así, existe una fuerte sensación dentro del sistema universitario europeo de que hay que "recuperarse" de un retraso, y ello pese a que el impulso de partida para crear este espacio común de educación superior tenía otras raíces.

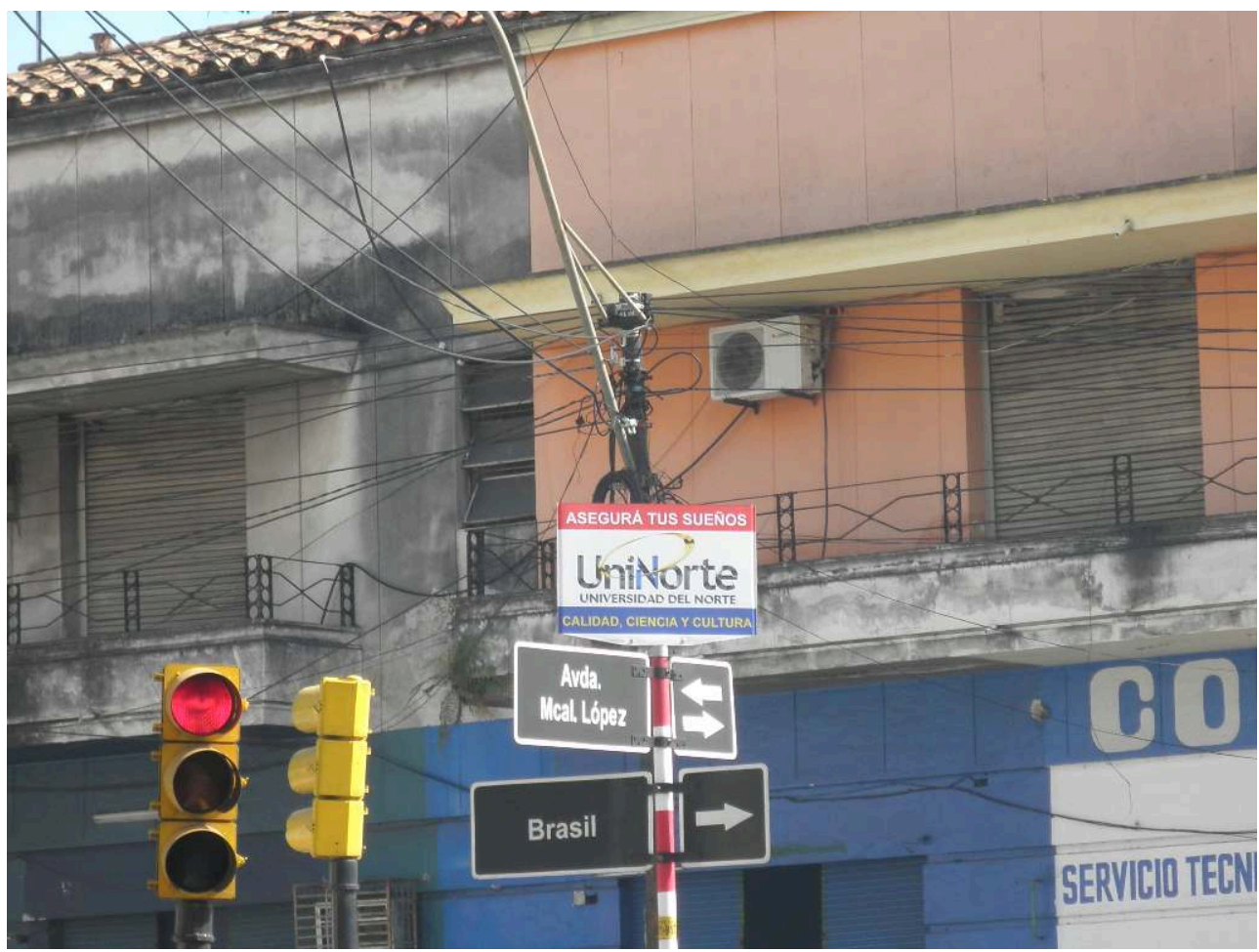

Publicidad de la universidad privada UNINORTE, omnipresente en Asunción (Paraguay): «Asegurá tus sueños», Universidad del Norte, Calidad, Ciencia y Cultura. El imperativo está reslatado según la pronunciación paraguaya influenciada por el idioma guaraní: "Asegurá".

Foto: C. Boidin, Paraguay, 2011

Recordemos que el proyecto de creación de un Espacio Europeo de Educación Superior se inició en la Sorbona en 19983: «La Europa que construimos no es solo la del Euro, los bancos y la economía; debe ser también una Europa del saber». El proceso fue lanzado oficialmente en 1999 en Bolonia y contó con la firma de 29 países. En la declaración de Bolonia, además, se añadió un objetivo ausente en 1998: «la cooperación europea en la garantía de calidad». ¿Qué significan estas dos palabras juntas: «garantía» y «calidad»? «En la educación superior, la garantía de calidad se puede conceptualizar en términos de las políticas, los procedimientos y las prácticas diseñadas con el objetivo de alcanzar, mantener o mejorar la calidad, tal y como se concibe en un contexto determinado»" En este dispositivo, la autonomía y la responsabilidad financiera de los centros educativos, así como el carácter externo de la evaluación, son los dos pilares que permiten «tranquilizar» a los 
diferentes socios en cuanto a la calidad de sus centros universitarios. En 2007, la creación del Registro Europeo de Garantía de Calidad (EQAR) precipitó la creación de estructuras nacionales de evaluación de la calidad en las universidades: en Francia, por ejemplo, se creó la AERES (Agencia de Evaluación de la Calidad de la Investigación y la Educación Superior).

Así, las universidades son consideradas entes gobernables a modo de empresas: por el método del benchmarking (elaboración de objetivos cuantificables que es necesario alcanzar urgentemente), descifrado por Isabelle $\mathrm{Bruno}^{5}$, se insta a individuos y organizaciones a someterse a estos objetivos, estableciendo la competencia entre ellos. La financiación de «iniciativas de excelencia», o por decirlo más claro, la financiación «específica» de instituciones y programas entra en esta lógica competitiva. Poner en marcha la cooperación puede resultar complejo y llevar a cabo cualquier reflexión sobre el fondo se vuelve imposible: la carrera por la financiación, por los objetivos y por la entrega de informes monopolizan toda la energía y llenan las agendas de las instituciones de educación superior. Como resume Boaventura de Sousa:

«In a few decades the university went from producing knowledge and professionals for the market, to becoming itself a market, the market of tertiary education, and finally, at least according to powerful visionaries, to being run like a market organization, a business organization.»

El segundo y último gran objetivo añadido al proyecto inicial definido en la Sorbona -el de la «dimensión social de la educación superior»- no se definió claramente hasta 2007 en el Comunicado de Londres en el que se declara, en último lugar, que «el cuerpo estudiantil que accede, participa y completa la educación superior a todos los niveles debería reflejar la diversidad de nuestras poblaciones» ${ }^{6}$. Con todo, en 2010 el balance es más modesto en este ámbito que en el de la creación de un marco común de referencia ${ }^{7}$ o en la implantación de la garantía de calidad, en atención a los criterios de buen gobierno (objetivos numéricos y por tanto, medibles):

«Muy poco países han establecido objetivos numéricos concretos para mejorar la participación de los colectivos infrarrepresentados en la educación superior, y solo la mitad de los países de Bolonia controlan de forma sistemática sus niveles de participación. Las medidas nacionales más comunes dirigidas a aumentar la participación consisten en ofrecer ayudas económicas para colectivos específicos y desarrollar itinerarios de acceso y/o procedimientos de admisión alternativos. (...) Lo más habitual entre los países firmantes del proceso de Bolonia es que la infrarrepresentación esté vinculada con el entorno socioeconómico, el nivel educativo de los padres, la pertenencia a minorías o la discapacidad. (...) Los diferentes enfoques utilizados para identificar a los colectivos infrarrepresentados demuestran lo sensible que puede ser este tema, de modo que cualquier comparación paneuropea resulta, en la práctica, inabordable». (las cursivas son nuestras)

En otras palabras, se establecen ayudas económicas específicas dirigidas a miembros de categorías concretas para permitirles el acceso a ciertas instituciones elitistas que pueden resultar competitivas a nivel internacional, disminuyendo de este modo la inversión en el resto de instituciones y reforzando el proceso de marginalización que se supone debería ser corregido por esta política. La panacea sería una universidad de élites «diversificadas» gracias a itinerarios de acceso alternativos. El objetivo marcado es reflejar la diversidad de la población, no promover la igualdad de oportunidades para todos. 
Para terminar, desde 1999 se han impulsado nuevos procesos de racionalización en las universidades europeas: multiplicación de criterios numéricos y «evaluables» para medir, clasificar y financiar la actividad de las universidades -tanto de la enseñanza como de las publicaciones- y búsqueda de la «diversidad» como criterio de justicia social. Estos dos dispositivos, de naturaleza diferente, caracterizan el funcionamiento del modelo norteamericano de educación superior pero en el fondo son el resultado de una historia singular.

\section{Más allá del modelo o del contramodelo: el sistema de educación superior estadounidense en perspectiva}

Pese a ser un modelo a seguir para unos y un contramodelo para otros, el sistema de educación superior estadounidense es poco conocido. Por ello y para abrir este dossier, es preciso recordar algunas de sus características principales. Formados durante el periodo de la América colonial, los primeros colleges, que por su antigüedad venerable se convertirán más tarde en las universidades de la Ivy League, son centros privados. Su función original es formar a las élites políticas y espirituales de las colonias británicas. La división territorial en trece colonias relativamente autónomas explica la multiplicación de estas instituciones que, tomando como modelo a Oxford y Cambridge, desarrollan un sistema de campus residencial para sus estudiantes. Una vez adquirida la independencia, la joven República reconoce inmediatamente la necesidad social de dotarse de instituciones públicas de educación en cada uno de los nuevos Estados creados (Ordenanza del Noroeste de 1785). Si bien en aquella época se trataba ante todo de implantar un sistema de escuelas públicas elementales, desde el principio se instaura el doble principio de la responsabilidad social de los Estados en materia de escolaridad pública y de su utilidad para la sociedad antes de ampliarse a todo el sistema de educación. Muy pronto se implanta un sistema múltiple que combina centros totalmente privados con otros financiados por el Estado federal donde se ubican. En efecto, una característica importante del sistema estadounidense es que la educación es competencia de los Estados federales, no del Estado central.

El periodo de 1785 a 1860 se caracteriza por una innovación extrema y un fuerte desarrollo, casi sin la más mínima supervisión o reglamentación por parte del Estado federal. Con todo, no se trata de un periodo caótico sino más bien de multiplicación de centros y de innovación disciplinar. Así, en todo el país, de 25 centros que en 1785 otorgan títulos superiores se pasa a 241 en 1860. Además de la creación de nuevos colleges, en este periodo asistimos también a la aparición de otro tipo de instituciones: las universidades propiamente dichas, las academias, los seminarios, las escuelas científicas, las escuelas normales y los institutos. Paralelamente a esta expansión, el programa que se enseña en los colleges se amplía de vez en cuando más allá de las liberal arts (combinación de latín, griego, matemáticas, además de las ciencias, la filosofía, la historia, así como la redacción y la literatura inglesa) para incluir la medicina, el derecho, la ingeniería, la ciencia militar, el comercio, la teología y la agricultura. 


\section{Nacimiento de las universidades públicas y las universidades reservadas a las minorías (mediados del siglo XIX)}

En la segunda mitad del siglo XIX asistimos a un doble fenómeno: por una parte, el afianzamiento de la red de centros de educación superior (con el nacimiento de nuevas universidades públicas y de colleges y universidades urbanas), y por otra, la creación de instituciones especialmente destinadas a permitir el acceso a la educación superior a grupos excluidos hasta entonces: mujeres y negros, por ejemplo.

Cabe mencionar para empezar la ley Morrill de 1862, todo un hito al oficializar la creación de los llamados land-grant colleges, esto es, centros de educación superior construidos en tierras concedidas por el Estado federado para este fin. Gracias a esta ley ven la luz las principales grandes universidades públicas del país. Es una de las grandes etapas de la democratización de la educación superior, a partir de ahora, de fácil acceso (tanto por el gasto poco elevado de los estudios como por la proximidad geográfica) para un gran número de jóvenes, y en especial, de chicas.

Mientras los land-grant colleges y las universidades de Estado permiten a jóvenes de numerosas regiones rurales y pequeñas ciudades acceder por fin a la educación superior -gracias a su proximidad geográfica y a su coste razonable- en las grandes ciudades surge un movimiento similar con la creación de colleges y universidades municipales. En efecto, también en los centros urbanos la tendencia es a construir centros públicos gratuitos o cuasi gratuitos ${ }^{8}$. El objetivo es responder a las necesidades de los estudiantes de las ciudades, en particular ofreciendo innovadores cursos de formación técnica e industrial avanzada o comercial, aparte de los cursos clásicos de liberal arts. Este tipo de centros permite por primera vez a los estudiantes de las ciudades y sin medios para matricularse a tiempo completo en un college residencial (con campus) cursar sus estudios y seguir viviendo con sus padres y/o trabajando gracias al desarrollo de la escuela nocturna y a los dispositivos para los estudiantes a tiempo parcial. Poco onerosos, estos colleges urbanos de proximidad consiguen ocupar pronto un lugar único e indispensable en el panorama de la educación superior en Estados Unidos.

El acceso de las mujeres a la educación superior se acelera tras la Guerra de Secesión. Hasta entonces había estado limitado a los pocos «seminarios» o «academias» abiertos a mujeres. El proceso de democratización reviste dos formas: la creación de instituciones específicas, los coordinate colleges, separados aunque vinculados a los colleges masculinos (Radcliffe queda adscrito a Harvard, Barnard, a Columbia, y Newcomb, a Tulane en Luisiana) y, paralelamente, un movimiento de desarrollo de la educación mixta ${ }^{9}$. A finales del siglo XIX, aproximadamente tres de cada cuatro colleges y universidades del país dan acceso a las mujeres, a pesar de que el número de estudiantes mujeres solo representa el 2,5\% del total de jóvenes estadounidenses.

La lucha de las mujeres por el acceso a la educación superior coincide con el inicio del movimiento de ampliación de oportunidades educativas para los negros. Antes de la Guerra de Secesión, solo 29 estudiantes negros pudieron obtener un título de educación superior. Después de la guerra, la mayoría de las iniciativas son sobre todo de tipo caritativo o religioso. Hacia finales de siglo, fundaciones filantrópicas de grandes empresas y ricos bienhechores privados prestan su apoyo para construir colleges negros. En total, entre los años 1870 y 1880 se crean probablemente unos 200 centros privados o religiosos destinados a estos estudiantes, aunque la gran mayoría no son en realidad 
más que centros de educación secundaria que palían la falta de institutos para afroamericanos. Estos centros desaparecen pronto por falta de financiación suficiente. De forma implícita, se plantea aquí un problema fundamental para el acceso de los negros a la educación superior en la América de la segregación: la naturaleza de la educación impartida y su adaptación al nivel de estudios real de los jóvenes negros de finales del siglo XIX.

La intención original de los fundadores de los colleges negros es ofrecer a estos estudiantes una educación idéntica a la que reciben los blancos, es decir, una educación de tipo liberal arts. Los líderes de la comunidad negra consideran que los futuros ministros del culto, los abogados, los médicos, los dirigentes políticos y los hombres de negocios del país deben, al igual que sus homólogos blancos, entrar en contacto con las tradiciones académicas y rechazan la idea de una formación industrial y agrícola rudimentaria como alternativa realista. Sin embargo, dado que apenas existen escuelas públicas de educación elemental y secundaria para los negros del sur, el número de estudiantes es muy reducido. Solo las universidades de Howard y Fisk ofrecen en la práctica la formación clásica de tipo liberal arts de nivel universitario. Frente a esta situación, numerosos filántropos empiezan a defender una educación técnica mejor adaptada a las oportunidades económicas y sociales reales (muy limitadas) de los negros del sur. El modelo en este sentido es el Instituto Tuskegee, fundado por el educador Booker T. Washington en Alabama en 1881. La filosofía de este popular centro de formación básicamente agrícola inspira la creación de los land-grant colleges para estudiantes negros, que se multiplican en el sur tras la decisión Plessy contra Ferguson de la Corte Suprema de Estados Unidos en 1896 que oficializa el carácter constitucional de la segregación al afirmar el principio de «separados pero iguales»: a partir de entonces, para no tener que aceptar a negros en las escuelas y en las universidades públicas de sus Estados, los gobiernos del sur se apresuran a crear colleges separados pero dotados en teoría de los mismos recursos y de fondos distribuidos de manera «justa y equitativa». Sin embargo, para la comunidad negra, la realidad de la situación es la de la separación, no la de la igualdad de trato. La democratización de la educación superior seguirá siendo en gran parte una quimera hasta la Segunda Guerra Mundial.

A pesar de estas importantes limitaciones, la democratización se ha iniciado y no se detendrá. Paralelamente a estos avances, a finales del siglo XIX tiene lugar la reestructuración de la educación superior como resultado de dos importantes factores. En primer lugar, la industrialización y el rápido desarrollo económico del país dan a los nuevos saberes técnicos y científicos una mayor importancia en la sociedad. La creencia de que la universidad debe modernizarse para responder a las necesidades del mundo económico se generaliza. En segundo lugar, es también la época en que llega a Estados Unidos el modelo universitario alemán -reconocido como el mejor de Europafocalizado en la investigación científica. Dentro de la educación superior estadounidense, esta popularidad se traduce en la creación de una nueva institución: la universidad de investigación, donde los docentes son ante todo investigadores cuya independencia debe preservarse y donde se desarrollan estudios de tercer ciclo, a diferencia del simple college, centrado en la enseñanza y la formación de tipo undergraduate (bachiller) y carente de una auténtica actividad de investigación. Este nuevo tipo de instituciones seduce especialmente a los nuevos centros urbanos, como la Universidad Johns Hopkins que, creada completamente a partir de este modelo, ofrece únicamente estudios de nivel graduate (licenciatura). Es por tanto a finales del siglo XIX y principios del XX cuando surgen en Estados Unidos las grandes universidades de 
investigación y un reducido entramado de centros especializados en estudios undergraduate de tipo liberal arts o semiprofesional. Esta división del panorama de la educación superior en Estados Unidos se mantiene hasta la actualidad.

\section{El impacto del G.I. Bill en 1945: masificación y evaluación}

Mientras prosigue la democratización de la educación superior en la primera mitad del siglo XX, especialmente con un acceso cada vez mayor de estudiantes judíos (las cifras empiezan a ser significativas en los años 1910, a pesar del establecimiento de pruebas y cupos específicos) y de mujeres (cuya proporción en el número total de undergraduates supera el 47 \% en 1920), el gran giro lo marca la ley conocida como GI Bill, de 1944, que garantizaba a los ex combatientes un año de estudios superiores gratis por cada 3 meses pasados en el ejército, y ello hasta un máximo de 48 meses. En el marco de esta ley, los gastos de matrícula y de otro tipo, así como la compra de libros y material, se abonaban directamente al college o a la universidad por un total de 500 dólares anuales como máximo (en una época en que los gastos de matrícula de las instituciones privadas ascendían a unos 300 dólares y los de las universidades de Estado eran considerablemente menos elevados). Además, los estudiantes recibían dietas. El éxito de la ley fue inmediato: a partir de 1946, en este contexto, el número de matrículas en la universidad supera el millón y las ayudas del gobierno federal ascienden a más de 5 500 millones de dólares. En 1950, de los 14 millones de ex combatientes que podían beneficiarse de esta ayuda, más de 2 millones eligieron proseguir sus estudios superiores.

El GI Bill constituye una novedad importante en la historia de la educación superior de Estados Unidos por varias razones: en primer lugar, esta ley hace de la continuación de los estudios superiores un derecho y no solo un privilegio como había sido hasta entonces. En otros términos, no hay límite en el número de beneficiarios. Además, este derecho, al ser concedido al estudiante, es "transferible" en el sentido de que un veterano puede solicitar su inscripción en Harvard pero también en un community college cercano a su domicilio, en una facultad de derecho o de medicina, o en una escuela de ingeniería. Para evitar cualquier abuso del sistema, el centro debe ser reconocido por el gobierno federal, lo que entraña, como consecuencia indirecta del GI Bill, la implantación de un sistema de evaluación de universidades. Preocupadas por mantener su independencia frente al gobierno federal, y considerando la $10^{\mathrm{a}}$ Enmienda a la Constitución de Estados Unidos, según la cual, «los poderes que la Constitución no ha delegado a los Estados Unidos, ni prohibido a los Estados, quedan reservados a los Estados respectivamente o al pueblo», las universidades optan por someterse voluntariamente a instancias autónomas de evaluación. Es el inicio del sistema de acreditación regional y de su ciclo de evaluación institucional cada diez años, que progresivamente se convierte en el procedimiento estándar para todas las instituciones que otorgan títulos, en especial si desean beneficiarse de las ayudas federales. Otro cambio inducido por el GI Bill es la manera en que las universidades y los colleges evalúan las candidaturas de los futuros estudiantes: ante la afluencia de solicitudes, la decisión de admisión debe tomarse ahora rápidamente. Con todo, dicha decisión es tanto más difícil de tomar cuanto que los ex combatientes casi nunca disponen de expediente académico, como sí tenían los estudiantes normales. Se implanta así un sistema de admisiones que recurre cada vez más a pruebas estandarizadas y que toma en consideración también otras experiencias diferentes a las adquiridas en el instituto. 
Otra consecuencia importante del GI Bill es la ampliación física de las universidades, que deben construir a toda prisa nuevos edificios para las clases, el alojamiento, los laboratorios y las instalaciones deportivas, pero también adaptar sus instalaciones a las necesidades específicas de los estudiantes veteranos, algunos de ellos, minusválidos. Igualmente asombrosa para el observador de la época es la transformación de la cultura estudiantil bajo el efecto de la llegada masiva de esta nueva cohorte: más mayores que los estudiantes de siempre, de entre 17 y 21 años, los que llegan a la universidad gracias al GI Bill tienen además una relación más pragmática hacia los estudios, con lo que las universidades se apresuran a diversificar su oferta para responder a su deseo de adquirir una formación que les permita obtener inmediatamente un empleo en la economía de la posguerra, en plena expansión. Su llegada supone también la masculinización del campus (a pesar de que unas 60000 ex combatientes también se beneficiaron del GI Bill) tras los avances registrados en décadas precedentes: habrá que esperar a los años 70 para que las mujeres representen de nuevo el $40 \%$ de los estudiantes undergraduate del país. Esta proporción asciende al $55 \%$ en la actualidad.

Con todo, el considerable impacto del GI Bill no debe exagerarse ya que conlleva una limitación fundamental: su escasa influencia en las relaciones raciales en los colleges y las universidades. Es cierto que había GI negros elegibles y que el número de estudiantes afroamericanos aumentó considerablemente gracias a este dispositivo, pero el texto de la ley no contemplaba ninguna obligación de no discriminación para las instituciones participantes. Los centros que tradicionalmente habían excluido a las minorías raciales siguieron haciéndolo con total impunidad (el mismo gobierno federal practicó hasta 1948 la segregación en las fuerzas armadas). A pesar de los avances democráticos del GI Bill, la doctrina del «separados pero iguales» siguió guiando las relaciones raciales tanto en la universidad como en el resto de instituciones del país. Esta situación perduró hasta la adopción por el Congreso, en 1964, de la gran Ley de Derechos Civiles, que prohibía la segregación y la discriminación que se practicaba todavía a pesar de la decisión de la Corte Suprema Brown contra el Consejo de Educación de Topeka, Kansas, de 1954.

Con todo, el GI Bill debe considerarse como el inicio de un nuevo papel en la educación superior asignado al gobierno federal que sigue existiendo en la actualidad. En 1947, el presidente Harry Truman encarga un informe, publicado bajo el título Higher Education for American Democracy, que subraya la importancia nacional de la educación superior en el nuevo contexto de la Guerra Fría, tanto desde el punto de vista social como de la influencia de Estados Unidos en el mundo y de la defensa del país. Esta visión apenas ha cambiado en 65 años, al contrario, incluso se ha reforzado valiéndose del aumento espectacular del número de universidades estadounidenses desde la Segunda Guerra Mundial.

Tras el conflicto, la educación superior en Estados Unidos goza de treinta años gloriosos de prosperidad, prestigio y popularidad. El gobierno federal prosigue su política de financiación de la investigación científica -cuya importancia se considera estratégica para el país- iniciada con el proyecto Manhattan para la fabricación de la bomba atómica durante la guerra. El gobierno comienza a financiar programas de investigación y formación en lenguas extranjeras y diferentes áreas culturales, y firma contratos de investigación con un importante grupo de universidades del país, encargadas de llevar a cabo por cuenta de aquél diversas misiones de investigación aplicada. Cabe recordar aquí que este dinero federal no sustituye en ningún caso a la 
contribución financiera de los Estados federados, que siguen siendo la principal instancia gubernamental encargada de los asuntos y la financiación educativa a todos los niveles. Sin embargo, no puede negarse que en las instituciones beneficiarias de estos contratos, el maná federal se volvió pronto indispensable para su funcionamiento, expansión y reputación, y que la inmensa mayoría de los colleges y universidades que no recibían esa ayuda se vieron en la obligación de buscar otras fuentes de financiación y otros medios de diferenciarse de la competencia para atraer a un número creciente de estudiantes deseosos de elegir su universidad en función de sus intereses y necesidades propias. Además de ofrecer un nivel de financiación inédito en la historia de la educación superior, esta nueva implicación del gobierno federal también condujo al aumento de la competición entre centros, que se vieron forzados a desarrollar especializaciones y una identidad única.

Paralelamente a esta función de financiación de la investigación por razones de interés nacional, el gobierno federal reconoce la doble necesidad de abrir las puertas de la educación superior a una mayor proporción de la población (necesidad inscrita en la importante ley sobre educación superior de 1965 que crea, entre otras, las becas de estudiante) y de dotarse de centros reconocidos en todo el mundo por la excelencia de su investigación y de su formación. El prolífico panorama universitario de la pre-guerra se refuerza de este modo con la multiplicación de los community colleges -centros de proximidad que imparten una formación semiprofesional por un coste muy razonableque amplían la base del sistema, mientras la cúspide de la pirámide sigue creciendo gracias a la capacidad demostrada de los colleges y las universidades para dotarse de programas avanzados selectivos a todos los niveles, siendo presentada esta selección como garantía de calidad. Paralelamente, aparece también en este periodo un nuevo tipo de institución: los centros privados con ánimo de lucro que ofrecen una formación especializada o profesional, y las llamadas corporate universities, creadas por iniciativa de grandes empresas.

En esta edad de oro basada en la democratización de la educación superior y el poder de la universidad de investigación se fundamenta, aún hoy, el prestigio del modelo de educación superior estadounidense. Otro factor apunta sin duda también a la capacidad de adaptación del sistema al doble desafío de la masificación provocada por el baby boom (se pasó de menos de 1,5 millones de estudiantes antes de la Segunda Guerra Mundial a 7,9 millones en 1970) y de las reivindicaciones de los movimientos identitarios de los años 60 y 70 (en particular, de las minorías etnoraciales y sexuales) que transformaron profundamente la universidad.

De hecho, las estructuras de educación superior en Estados Unidos apenas han evolucionado desde los años 70, a pesar de que han surgido nuevos desafíos con la crisis económica de 2008. Dicha crisis ha llevado a una considerable disminución de los recursos disponibles para la educación superior (y para la educación en general) en el presupuesto del Estado federal y de los Estados federados, provocando un nuevo aumento de los derechos de inscripción y dejando la educación superior fuera del alcance de una parte creciente de la población. Este peligroso cuestionamiento de décadas de democratización del acceso a la educación superior plantea actualmente una cuestión inimaginable hasta ahora: la de los límites del modelo americano y su durabilidad. Es en este contexto donde conviene interpretar el llamamiento del presidente Obama a relanzar el desarrollo de la educación superior en Estados Unidos, 
apostando no ya por la excelencia de las instituciones de élite como motor del sistema, sino por los community colleges ${ }^{10}$.

En un país donde la población estudiantil ascendía a 19,1 millones en 2010 (frente a 13,8 millones en 1990, lo que representa actualmente más del $41 \%$ de las personas entre 18 y 24 años), repartidos en 4352 centros, y donde el coste de los estudios se ha duplicado con creces desde 1990 (en 2010, el coste medio anual de los gastos de matrícula y alojamiento en los colleges y universidades públicas para los estudiantes del Estado es de casi 15000 dólares frente a poco más de 40000 en los centros privados), se entiende bien este interés en reorientar las prioridades nacionales, un hecho que sin duda debería analizarse también en otros países.

Paradójicamente, la implantación de sistemas estandarizados de evaluación se remonta a los años 1945 con el GI Bill, que fue un incentivo para la democratización del sistema. La racionalización de dicho sistema se impuso para hacer frente a su crecimiento numérico y controlar la circulación de fondos públicos. En realidad, el sistema de educación superior norteamericano funciona, de entrada, a varias velocidades y se basa en la multiplicación de instituciones especializadas (ya sean universidades o departamentos) para integrar a diferentes sectores de la población. El artículo de Caroline Rolland da al lector una visión del desarrollo experimentado por los departamentos de estudios negros (black studies) en Estados Unidos en los años sesenta. Ramón Grosfoguel, por su parte, analiza los dilemas actuales de los departamentos de estudios étnicos (ethnic studies), dilemas que comparten en gran medida las universidades indígenas de México (artículo de David Lehmann) y Bolivia (artículo de Anders Burman), aunque parten de una historia universitaria diferente cuyas raíces se remontan a la España medieval.

\section{El sistema latinoamericano de enseñanza universitaria en el cruce de los modelos europeo y estadounidense}

A modo de comparación con el proceso norteamericano, cabe presentar aquí varias referencias históricas ${ }^{11}$. Antes de 1492 pueden encontrarse en fuentes sobre los imperios inca y azteca instituciones especializadas en la formación académica de las élites. Con la conquista española se difunde y adapta el modelo de universidad medieval, escolástico, que en España encarnan Salamanca (Studium Generale, 1218) y Alcalá de Henares (Estudio de escuelas generales, 1293). Inicialmente, las clases se imparten en el claustro de la catedral y en casas arrendadas de las ciudades. A principios del siglo XV se construyen los colegios (edificios que incluyen un internado para los estudiantes pobres y becarios, además de aulas y otros espacios para actividades sociales, culturales y deportivas). Los colegios menores eran los que daban el título de bachiller y los colegios mayores los que dispensaban estudios de licenciatura y doctorado, aunque los exámenes se hacían en la universidad. Con el tiempo, los colegios son monopolizados por la nobleza. La influencia de Salamanca en particular se aprecia en Lima (1575) y México (1595) a través de la adopción de sus modos de organización, sus programas, sus prácticas, sus privilegios y la circulación de sus docentes. Ambas compiten en cierto modo para destacar sobre el resto de universidades regionales. La mayoría de las universidades reciben autorizaciones, a la vez reales y pontificias. Con frecuencia, se establecen en seminarios, conventos o en los colegios de dominicos, agustinos o jesuitas ${ }^{12}$. A algunas universidades se las considera 
mayores, oficiales y generales, con rentas de la real hacienda y numerosas cátedras y privilegios. Otras se consideran particulares, al no poseer las mismas facultades para otorgar diplomas. Las universidades se organizan en 4 facultades mayores (Teología, Cánones, Leyes y Medicina) y una facultad menor de Artes o Filosofía. Para las élites indígenas de México se creó muy pronto un colegio de franciscanos, llamado Santa Cruz de Tlatelolco (1533). Durante unos cincuenta años, la enseñanza del latín y la utilización del alfabeto latino para el náhuatl dieron pie al nacimiento de importantes obras científicas basadas en conocimientos médicos e históricos nahuas. Las primeras generaciones de indígenas formados en colegios tuvieron importantes responsabilidades en su propia universidad y en otras instituciones coloniales antes de que el modelo quebrara. Este modelo difusionista, que insiste en la importación del modelo medieval español, es demasiado simplista pero, con todo, da cuenta de un hecho importante: la fundación rápida y perdurable de veinticinco universidades en todo el territorio de la América española mientras la corona portuguesa no permitió la apertura de colegios, y no de universidades, en suelo brasileño hasta 1808 , fecha en la que la familia real se instala en Brasil.

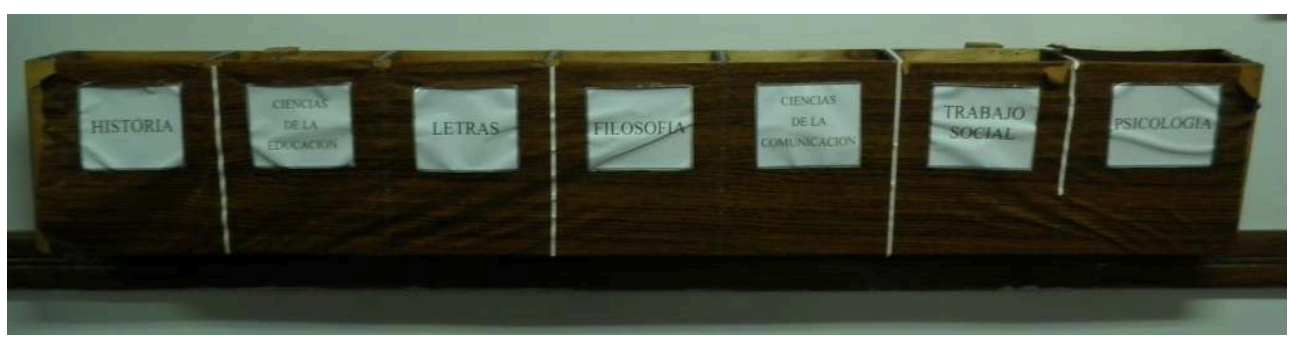

UNA HISTORIA CONCRETA PARA ILUSTRAR LA CONSTRUCCIÓN DEL SABER: BUZONES DE LOS SIETE DEPARTAMENTOS DE LA FACULTAD DE FILOSOFÍA DE LA UNIVERSIDAD NACIONAL DE ASUNCIÓN (PARAgUAY).

FOTO: C. BOIDIN, PARAgUAY 2011

Los aires de la Ilustración francesa soplan con fuerza en las universidades latinoamericanas a finales del periodo colonial, antes de que la etapa de independencias republicanas acabe difundiendo los principios de organización napoleónicos (largas carreras profesionales de entre 6 y 7 años, sin títulos intermedios) y el principio humboldtiano de vincular la enseñanza a la investigación. El positivismo comtiano también tuvo un importante impacto tanto en el ámbito hispano como en el de habla portuguesa. En el siglo XX, como recuerda el artículo de Guadalupe Olivier en este dossier, se dan tres momentos clave: la Reforma de Córdoba de 1918, que defendió el principio de la gratuidad de los estudios y la lucha por la democratización de una institución concebida como pública y autónoma; la reforma de los años 70, que supuso el crecimiento de instituciones superiores muy diferenciadas que no necesariamente eran de tipo universitario pero que permitieron dar mayor cobertura al sistema público, y la tercera reforma que, desde los años noventa, supone la masificación, la privatización y la internacionalización de la educación superior latinoamericana. 


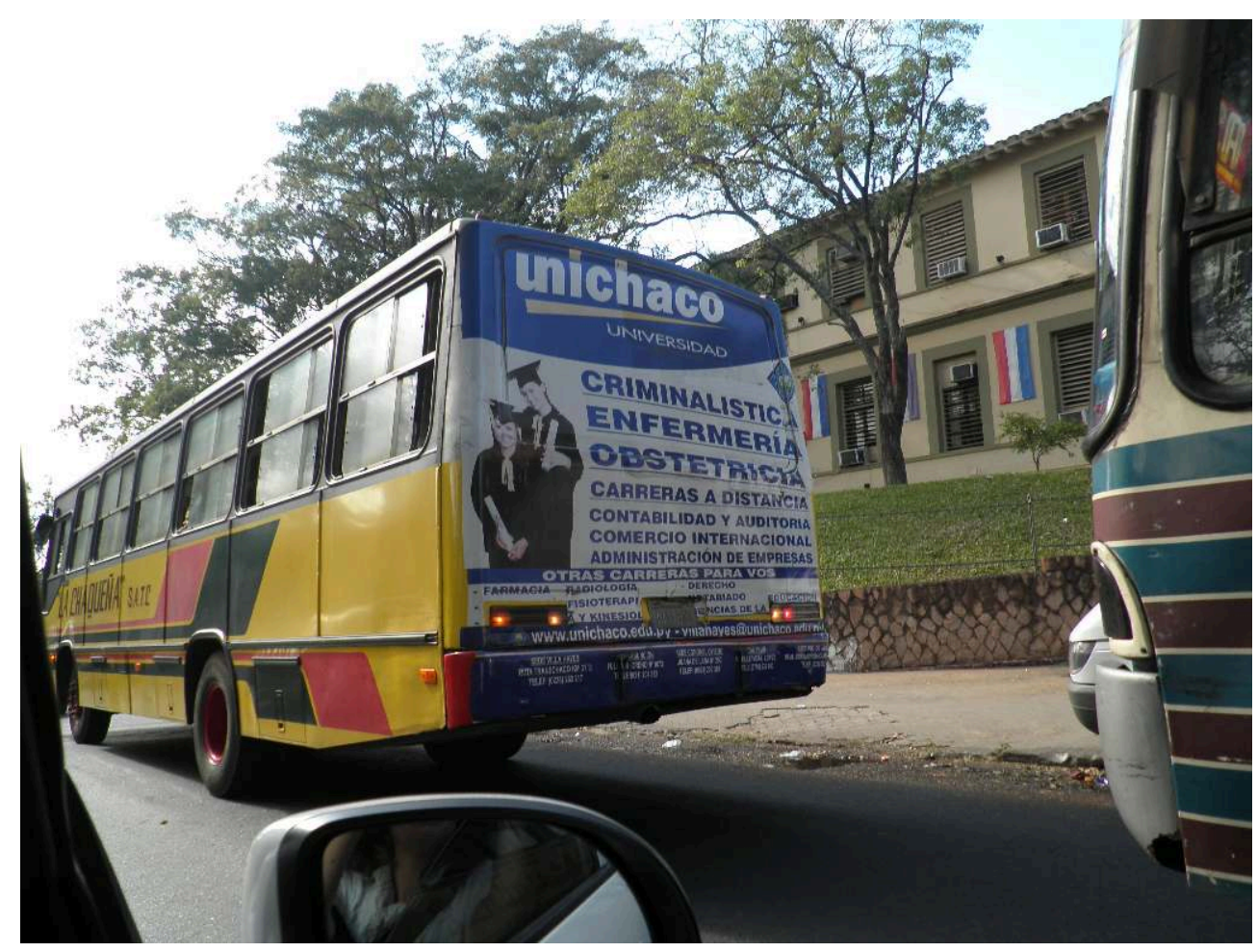

Abundante publicidad de universidades privadas en América Latina. Asunción, Paraguay, 2011.

FOTO: C. BOIDIN 2011

\section{Masificación, privatización y desarrollo de universidades indígenas en la educación superior de América Latina}

En este ámbito se oponen dos tipos de análisis divergentes: unos, basados en una larga tradición de pensamiento crítico en América Latina, descifran las políticas neoliberales contemporáneas ${ }^{13}$, y otros multiplican los informes de síntesis para justificar sus "recomendaciones" en materia de reformas (Banco Mundial y Fondo Monetario Internacional, en particular). Antes de proseguir, he aquí algunas cifras relativas a la aceleración de la masificación y la privatización en el sistema de educación superior latinoamericano ${ }^{14}$ :

Número de universidades e instituciones de educación superior (IES) en América Latina

195075 universidades

1975330 universidades

1985450 universidades

1995812 universidades (60,7 \% privadas) 5438 IES

20021213 universidades (69,2\% privadas) 7514 IES

Actualmente: más de 2000 universidades, probablemente

Número de estudiantes inscritos en el sistema de educación superior de América Latina

1950267000

19701640000

19804930000

19907350000 
200011500000

200515293181

200917757000

Actualmente (2012): 18 millones aproximadamente

Brasil cuenta actualmente con 5,95 millones de estudiantes inscritos ${ }^{15}$ y México y Argentina, con unos 2,5 millones cada uno. Los tres países suman un total de unos 11 millones de inscritos, esto es, el $60 \%$ de los 18 millones de estudiantes latinoamericanos actuales. Son también los tres países con el mayor número de estudiantes de máster y doctorado. En número total de estudiantes les siguen Colombia (1,3 millón), Venezuela (1 millón), Perú (900 000), Chile (670 000), Cuba (470 000) y Bolivia (350 000). La tasa de matriculación en educación superior en América Latina ha mejorado considerablemente, pasando de $17 \%$ a principios de los años 90 a $34 \%$ en la actualidad. La situación varía notablemente de un país a otro: 18 \% en Guatemala, 27 \% en México, 30 \% en Brasil, 32 \% en Colombia, 35 \% en Perú, 52 \% en Venezuela, 67 \% en Argentina y hasta $88 \%$ en Cuba. Dicho de otro modo, aunque México y Argentina tienen el mismo número de estudiantes, el primero está lejos de cubrir las expectativas de la población como lo hace el segundo. Por otra parte, algunos sistemas educativos son particularmente desiguales: las familias con escasos recursos económicos quedan excluidas de las universidades públicas de calidad por no haber podido inscribir a sus hijos en buenas escuelas de secundaria (privadas o públicas). Es especialmente el caso de Brasil, pero también de México, Chile y Ecuador. La inversión anual global por estudiante latinoamericano es actualmente de 2380 dólares. En resumen, si bien en cifras totales de estudiantes, América Latina y Estados Unidos tienen curiosamente el mismo peso (18 y 19 millones respectivamente), la financiación en América Latina es considerablemente menor (recordemos que los gastos de inscripción en Estados Unidos oscilan entre 15000 y 40000 dólares anuales).

Esta masificación que algunos califican de democratización no se habría producido sin la multiplicación de universidades privadas, ya sean sin ánimo de lucro o claramente orientadas a la captación de la inversión educativa que cada vez más familias latinoamericanas están dispuestas a realizar por sus hijos. Los procesos de integración en las Américas suponen la creación de acuerdos de asociación entre instituciones de educación superior de diversos países, públicas o privadas (ver artículo de Guadalupe Olivier en este dossier ${ }^{16}$ ), y la multiplicación de estudios comparativos. 


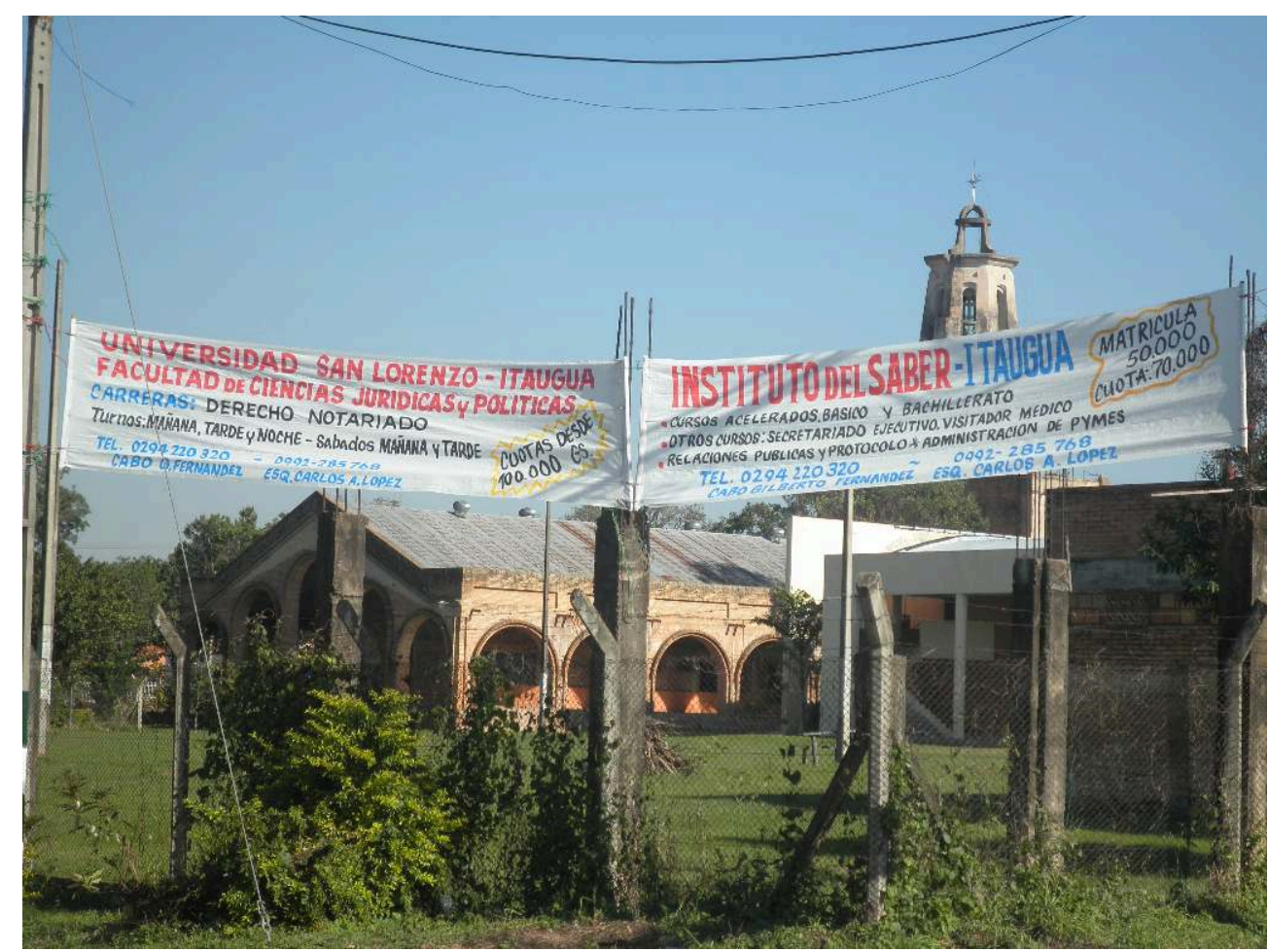

Publicidad de una universidad en un lado y de un «Instituto del saber» en el otro, San Lorenzo, Paraguay.

Foto: C. Boidin 2011

Desde los años 60, diversos organismos de la UNESCO, además de la CEPAL (Comisión Económica para América Latina y el Caribe) han impulsado estudios comparativos de los sistemas de educación a escala latinoamericana. A partir de 1978, el Centro Regional para la Educación Superior en América Latina y el Caribe (CRESALC), transformado a finales de los años 90 en el Instituto Internacional para la Educación Superior en América Latina y el Caribe (IESALC), ha elaborado numerosos informes, tales como el Documento de política para el cambio y el desarrollo de la educación superior, en 1995, que llevó a la UNESCO a definir, en 1998, un plan de acción organizado en torno a cinco ejes: 1) la pertinencia social de la enseñanza, 2) la calidad, la evaluación, 3) la gestión y la financiación, 4) la utilización de nuevos medios de comunicación y 5) la cooperación internacional. Estos cinco ejes recuerdan especialmente a los que fijó la Unión Europea en 1999, con la diferencia de que la dimensión social de la enseñanza se presenta como prioritaria por delante de la voluntad de evaluación.

Al final, algunos años antes que Europa, varios países de América Latina han liberalizado ampliamente su sistema de educación superior y han implantado sistemas estandarizados de evaluación de la investigación y la enseñanza, al tiempo que han multiplicado el número de instituciones destinadas principalmente a las poblaciones autóctonas: las universidades llamadas "indígenas" o "interculturales" en función de los países y los periodos, que estudian en este dossier Anders Burman, en el caso de Bolivia, y David Lehmann, para México. 


\section{Desprovincializar las universidades europeas}

En Francia, la reforma de las universidades, acelerada en 2007, suscitó grandes protestas y sacó a la calle a manifestantes de todo el espectro político, de derechas y de izquierdas. El deseo de defender una idea singular del saber científico y la educación superior considerados «bienes colectivos y públicos» y no «bienes de consumo individual» (un título válido y útil en la carrera del individuo) abarcaba tanto a las ciencias humanas y sociales como a las ciencias jurídicas, las ciencias físicas, las matemáticas y las ciencias naturales. Pasar de una relación pedagógica a una relación comercial en donde el estudiante se convierte en cliente es contrario a la cultura universitaria europea. En la opinión pública, en cambio, este tipo de defensa ha podido ser interpretada como el rechazo de los profesionales universitarios a preparar a sus estudiantes para integrarse en el mundo profesional. Y sin embargo, no se trataba de ir contra la integración profesional de los estudiantes: una formación rigurosa agudiza el sentido crítico y la autonomía de los estudiantes, preparándoles para adaptarse inteligentemente a cualquier situación social y, en particular, profesional. Se trataba más bien de reaccionar contra la importación de una cultura empresarial dentro de la universidad y la transformación de la universidad en empresa.

Al final, todos estos malentendidos y confusiones nos han llevado a preguntarnos si la crisis de las universidades francesas, o más generalmente europeas, no tiene su origen en las presiones generadas por el Proceso de Bolonia. Dicha crisis vendría provocada igualmente por el estancamiento del modelo universitario y del universalismo heredado de la época de las Luces. En realidad, las críticas internas hacia el conocimiento y las relaciones con el saber practicado en las universidades no son nuevas (Foucault, Bourdieu, Lyotard, Latour, Rancière, etc.). Con todo, desde hace unos diez años, el modelo de universidad kantiana-humboldtiana («la ciencia por y para la ciencia», desligada de la teología; carácter enciclopédico de la investigación; figuras del investigador-docente y del estudiante-investigador) es también ampliamente cuestionado y criticado por pensadores poscoloniales asiáticos, latinoamericanos, norteamericanos y europeos. En particular, los autores latinoamericanos -que prefieren llamarse descoloniales a poscoloniales- estudian el eurocentrismo epistémico que rige en ciertas prácticas de la institución. Denuncian el eurocentrismo, el racismo y el sexismo epistémico de instituciones en donde solo las ideas elaboradas a partir de las tradiciones occidentales y publicadas por europeos o euroamericanos son consideradas fuente de universalidad. Yendo más allá, cuestionan la voluntad total de saber enciclopédico, en particular antropológico, que, si bien parte del principio de conocer a los «otros», no conduce a su pleno conocimiento como sujetos y autores. Ahora bien, esta crítica no lleva necesariamente a un relativismo estricto ni al abandono de la investigación universal. Por el contrario, el interés de las reflexiones más recientes de los pensadores latinoamericanos poscoloniales estriba en el hecho de subrayar la necesidad de un proceso de reflexión universal que se construya con el diálogo entre investigadores/as procedentes de mundos epistémicos plurales.

Precisamente, las reformas propuestas por el Proceso de Bolonia no afrontan estas profundas críticas. Al contrario, al centrarse esencialmente en los métodos de gestión y de evaluación de los docentes y los títulos, dichas reformas refuerzan el desencanto del mundo universitario. Con todo, el potencial de renovación de las universidades francesas y europeas es considerable, en particular, si se abren más decididamente a 
diálogos inter-epistémicos donde el reto consistiría en construir una nueva ecología de saberes. Lejos de limitarse a un relativismo blando y por defecto, o a "pequeños relatos", el objetivo sería buscar saberes universales pero pasando por la instauración de diálogos horizontales entre las distintas tradiciones de pensamiento. Reformar la universidad buscando un universalismo menos provincial y más abierto implica un cambio profundo en nuestra manera de pensar y en nuestras divisiones disciplinares. La creación del campus Condorcet podría brindar la ocasión de buscar formas de pensar pluri-versales y mantenerse en línea con las cuestiones formuladas por la sociedad ${ }^{17}$. Esta apertura y esta nueva construcción de saberes se hacen visibles en la arquitectura elegida para este campus. Las conversaciones con Cynthia Ghorra-Gobin y João Sette Whiteker Ferreira sobre la organización de los campus californiano y brasileño respectivamente permitirán comprender, comparativamente, las especificidades de este campus localizado en el norte del gran París y abierto a la cotidianeidad de barrios populares en plena reconstitución como Aubervilliers y Saint Denis.

Por esta razón, el presente dossier (y la conversación campus que le acompaña) pretende impulsar el diálogo con otras tradiciones de pensamiento, en particular latinoamericanas, $\mathrm{y}$ otras experiencias como las universidades indígenas latinoamericanas. En efecto, al tiempo que las corporate universities de Estados Unidos se erigen como modelo a partir de los años 70 , América Latina va a copiar rápidamente el modelo para multiplicar este tipo de instituciones en los años 80, abriendo también la posibilidad de que se creen las universidades autónomas e indígenas. Dicho de otro modo, el análisis y el debate sobre las transformaciones universitarias que tienen lugar desde hace cuarenta años en el continente americano, y en Europa, desde hace una década, deberían permitirnos una mejor comprensión de la situación que atravesamos para idear mejor la universidad europea del mañana.

\section{NOTAS}

1. Jugamos aquí con el sentido que Dipesh Chakrabarty da a la expresión "provincializar» en su obra Al margen de Europa: Pensamiento poscolonial y diferencia histórica, Tusquets Editores, Barcelona, 2008 (Traducción de Alberto E. Álvarez y Araceli Maira). Para Chakrabarty, que analiza la situación india, "el pensamiento europeo es a la vez indispensable e inadecuado para reflexionar sobre las experiencias de la modernidad política en las naciones no occidentales, y provincializar Europa se ha convertido en la tarea de explorar cómo este pensamiento -que ahora es patrimonio de todos y nos afecta a todos- podría ser renovado desde y para los márgenes. Pero, por supuesto, los márgenes son también tan plurales y diversos como los centros» (p. 53). Para nosotros, que reflexionamos desde Europa, desprovincializar las universidades europeas consiste en reconocer, humildemente, la necesidad vital de «conectarse», de renovarse a través de las más diversas tradiciones de pensamiento. Esta idea se explicita más ampliamente al final de este editorial.

2. Para una clasificación reciente de las universidades latinoamericanas, véase http://americalatina.blog.lemonde.fr/2011/10/05/l'universite-de-sao-paulo-en-tete-du-premier-classementlatino-americain/ 
3. http://www.cpihts.com/PDF/Declaracao\%20de\%20la\%20Sorbonne.pdf consultado el 2 de junio de 2012.

4. http://eacea.ec.europa.eu/education/eurydice/documents/thematic_reports/122FR.pdf

5. Isabelle Bruno, «Y a-t-il un pilote dans l'Union ? Tableaux de bord, indicateurs, cibles chiffrées : les balises de la décision», Politix, vol. 82, nº 2, 2008, p. 95-118.

6. Comunicado de Londres, 2007 (p. 5), citado en el informe de la Comisión Europea La modernización de la educación superior en Europa: financiación y dimensión social, 2011 (p. 9).

7. Generalización del sistema de tres ciclos (Grado, Máster, Doctorado) y acumulación de ECTS a lo largo de la carrera, independientemente de la universidad o la región.

8. Una tendencia no del todo nueva ya que la Free University of New York City se funda en 1847, por ejemplo. La tendencia se acelera tras la Guerra de Secesión cuando nacen la Universidad de Cincinnati, la Hunter College de Nueva York, la Wayne University de Detroit, la Universidad de Boston, la Temple University de Filadelfia o la Universidad George Washington de Washington.

9. En este sentido, juegan un papel pionero las nuevas universidades públicas, primero, las Universidades de Iowa (1855) y de Wisconsin (1863), seguidas de las Universidades de Indiana, Misuri, Michigan y California en los años 1870. A partir de esta década, la mayoría de los centros de educación superior del Oeste y del Medio Oeste aceptan esta práctica. La resistencia se concentra sobre todo en las Universidades del Ivy League del Noreste.

10. Como bien afirma Dominique Godrèche los community colleges «accesibles para todos, sin limitaciones de edad ni distinciones sociales $u$ origen académico, representan en Estados Unidos una alternativa a una enseñanza universitaria tan cara como selectiva. Al término de la escuela secundaria, estos estudios de dos años otorgan el associate degree, necesario para obtener después de dos años de universidad- el bachelor degree. De este modo, los estudiantes de las clases desfavorecidas, que no podrían pagarse cuatro años de estudios en una universidad privada, pueden acceder a la educación superior. Además, estos colleges dispensan una enseñanza profesional avalada por certificates (certificados académicos), además de una educación diversificada, a un sector de la población que, de otra forma, no tendría derecho a nada, como es el caso particular de los inmigrantes. [...] La integración de los community colleges en el tejido económico de sus regiones y su capacidad de reacción para adaptar los estudios a las nuevas necesidades constituyen para ellos importantes ventajas». Véase Dominique Godrèche, «En marge des universités, les community colleges» $\mathrm{y}$ «Onze millions d'étudiants», Le Monde diplomatique, junio de 2010.

11. Para un primer acercamiento, véase: Celina A. Lértora Mendoza, «Lineamientos para una historia de la universidad latinoamericana», Cuadernos de la Facultad de Humanidades y Ciencias Sociales, diciembre, $n^{\circ} 15$, pp. 43-51. Para profundizar, véase: Diana Soto Arango, Manuel Lucena Salmoral y Carlos Rincón (dir.), Estudios sobre la universidad latinoamericana. De la colonia al siglo XXI, RUDECOLOMBIA, HISULA, Universidad pedagógica y tecnológica de Colombia, Ed. Doce Calles, 2003. 12. Ver Águeda Rodríguez Cruz, La proyección de la Universidad de Salamanca en Hispanoamérica, 2012, consultado en http://www.americanistas.es/biblo/textos/s04/s-04-10.pdf y La Universidad en la América hispánica, Madrid, Mapfre, 1992.

13. Véase en particular los trabajos de Mollis, M. (comp.), Las universidades en América Latina: ¿reformadas o alteradas?, Buenos Aires, CLACSO, 2003.

14. Francisco López Segrera, «La educación superior en el mundo y en América Latina y el Caribe», Revista paraguaya de sociología, año 47, n 136, enero-junio de 2010. López Segrera es una de las principales referencias por sus informes de la UNESCO sobre la educación superior América Latina desde los años 2000. Cuando las cifras que se muestran no proceden de su artículo, lo señalamos.

15. http://gestao2010.mec.gov.br/indicadores/chart_60.php datos elaborados por el ministerio de educación superior de Brasil. 
16. http://america-latina.blog.lemonde.fr/2011/10/05/l\%E2\%80\%99universite-de-sao-paulo-entete-du-premier-classement-latino-americain/

17. Estos principios se formulan en el programa científico del Campus en estos términos: «Reunir las Humanidades y las Ciencias Sociales; combinar el estudio del pasado y el estudio del presente; promover las problemáticas transversales, adaptadas al análisis de las dinámicas de un mundo global. Estos principios están asociados a dos orientaciones: hacia los objetos (áreas culturales, religiones, textos, territorios, poblaciones, etc.) predilectos de las disciplinas, y hacia la creación de una herramienta para el conocimiento y la comprensión de lo contemporáneo que esté ligada a una ampliación y una renovación de las salidas en el ámbito de las ciencias humanas y sociales». http://www.campus-condorcet.fr/campus-condorcet/La-politique-scientifique/Le-programmescientifique/p-451-Le-programme-scientifique.htm

\section{AUTORES}

\section{CAROLINE ROLLAND-DIAMOND}

Maître de conférences en histoire à l'Université Paris Ouest Nanterre. Ses axes de recherche portent sur l'histoire sociale, l'histoire politique et l'histoire urbaine des États-Unis au XXe siècle, ainsi que sur l'histoire des mouvements étudiants américains depuis les années 1960, l'histoire de l'institution universitaire, la théorie des mouvements sociaux et l'histoire de la répression politique. Elle est l'auteur de Chicago : le moment 68 . Territoires de la contestation étudiante et répression politique (Paris: Syllepse, 2011) et a co-dirigé (avec Robi Morder) Etudiant(e)s du monde en mouvement. Migrations. Cosmopolitisme et internationales étudiantes (Paris, Syllepse, 2012).rolland.caroline@gmail.com

\section{CAPUCINE BOIDIN}

Maître de conférences en anthropologie à l'Université Sorbonne Nouvelle Paris 3, IHEAL (Institut des Hautes Etudes d'Amérique Latine). Chargée de cours de guarani à l'INALCO (Institut National des Langues et Civilisations Orientales), ses axes de recherche portent sur le métissage, le genre, les mémoires de guerre. Elle a publié en 2010 un numéro des Cahiers des Amériques latines sur le tournant décolonial.

http://www.ladocumentationfrancaise.fr/catalogue/3303332400621/index.shtml ;http:// www.iheal.univ-paris3.fr/spip.php?article1041

\section{JAMES COHEN}

Professeur à l'Institut du monde anglophone et enseignant à l'Institut des hautes études de l'Amérique Latine, Université de Paris 3 Sorbonne Nouvelle. Spécialiste des questions de race/ ethnicité et d'immigration aux Etats-Unis et du rôle des Etats-Unis dans les Amériques. jim.cohen@libertysurf.fr

\section{RAMON GROSFOGUEL}

Maître de conférences (Associate Professor) en études ethniques à l'université de Californie, Berkeley et chercheur invité auprès de la Maison des sciences de l'homme à Paris. Il a publié de 
nombreux articles et ouvrages sur l'économie politique du système mondial et sur les migrations caribéennes en Europe occidentale et aux États-Unis. Grosfogu@Berkeley.edu 